\title{
Prevalence of palate-gingival groove in patients of Dhulikhel Hospital
}

\section{Shrestha $D^{1}$, Humagain $M^{2}$, Swastika $S^{3}$}

${ }^{1}$ Lecturer, Department of Conservative Dentistry, ${ }^{2}$ Assistant Professor, Department of Periodontology, ${ }^{3}$ Dental Surgeon, Department of Conservative Dentistry Kathmandu University School of Medical SciencesBDS Program

\begin{abstract}
Background:

Palato-gingival groove is a rare developmental anomaly leading to pulpal and periodontal diseases. Radicular extensions of these grooves are areas for plaque accumulation which are difficult to clean. Its clinical and radiographic findings mimic endo-perio lesions leading to diagnostic dilemma, ineffective treatment attempts and treatment failures.
\end{abstract}

\section{Objectives:}

To investigate the prevalence of coronal and radicular palato-gingival groove in dental patients visiting Dhulikhel Hospital and its association with gingival disease.

\section{Methods:}

Patients ( $N=231$ ) visiting dental out-patient department of Dhulikhel Hospital, were examined for the presence or absence of palato-gingival groove in maxillary anteriors. Loe and Silness Gingival index was also recorded for all the patients using University of North Caroline-15 periodontal probe. Chisquare test was used to test the association of the presence of palato-gingival groove and gingival disease. $\mathrm{P}$ value of $<0.05$ was considered to be significant.

\section{Results:}

A total of 1362 maxillary anterior teeth were included in the study. The prevalence of coronal palatogingival groove among 1362 teeth was 6.6\%. Coronal palato-gingival grooves were more common in females $(56.6 \%)$ than in male $(43.3 \%)$ patients. Prevalence of radicular groove was only $0.88 \%$ for lateral incisors and $0.2 \%$ for canines. There was no significant asociation between palatogingival groove and gingival disease $(P=0.91)$.

\section{Conclusion:}

The prevalence of palato-gingival groove is common in dental patients visiting Dhulikhel Hospital. Gingival disease is not significantly associated with palate-gingival groove.

Keywords: Dhulikhel, palato-gingival groove, prevalence.

Correspondence: Dr. Shrestha D

E-mail: deeptishrestha@hotmail.com 


\section{INTRODUCTION}

Palato-gingival groove (PGG) or radicular lingual groove is a developmental anomaly in which an infolding of the inner enamel epithelium and Hertwig's epithelial root sheath create a groove that passes from the cingulum of maxillary incisors apically onto the root. ${ }^{1} \mathrm{The}$ funnel-like shape of the palato-gingival groove promotes the accumulation of difficult-to-remove plaque and calculus, at times making proper cleaning by the patient and dentist, nearly impossible. ${ }^{2}$ Moreover, a direct communication between the pulp and the periodontium can lead to pulpal diseases. ${ }^{3}$ As a microscopic analysis is required to analyze the communication between the pulp and periodontium, it can lead to diagnostic dilemmas as they mimic endoperio lesions. ${ }^{4}$

Various in vivo and in vitro studies have shown a prevalence of less than two to $11 \%$ for PGGs. ${ }^{3,5-11}$ More plaque, bleeding, probing depths and caries incidence were found in teeth with the groove. ${ }^{9-12}$ Till date, no study has been done to investigate the prevalence of palato-gingival grooves in Nepalese population.

This study investigated the prevalence of coronal and radicular PGGs in dental patients of Dhulikhel Hospital. It further investigated the association of PGGs and gingival disease.

\section{METHODS}

This cross-sectional observational study was conducted in Dental out-patient department (DOPD) of Dhulikhel Hospital from June 2013 to July 2013. Two hundred and thirty one patients ( 12 years to 60 years old) visiting the DOPD, Dhulikhel Hospital, were randomly chosen to participate in this study.
The examination site was dried using cotton pellets. A mouth mirror, an explorer and magnifying loupes $(\times 1.5)$ were used for inspection. Maxillary central incisors (CI), lateral incisors (LI) and canines $(\mathrm{CN})$ were investigated for the presence or absence of PGG. In the presence of PGG, it was recorded as coronal (coronal to cement-enamel junction) or radicular (apical to cemento-enamel junction). The site of PGG was also recorded as mesio-palatal, mid-palatal or distopalatal. Gingival index (Loe and Silness, 1963) of individual tooth was recorded using a University of North Caroline-15 (UNC-15) periodontal probe (HuFriedy, Chicago, IL). ${ }^{13}$ Teeth with gingival index score of less than one were categorized as no gingival disease, while those with more than or equal to one were categorized as gingival disease.

The study was approved by the Institutional Review Committee of Kathmandu University School of Medical Sciences. A written consent in Nepali language was obtained from all the patients participating in the study.

Any teeth with dental caries, direct or indirect restorations, rotation, impaction or congenital malformations were excluded from the study. Chisquare test (level of significance set at 0.05 ) was done using statistical package for social sciences (SPSS) (version 16.0; SPSS Inc, Chicago, IL). The null hypothesis stated PGGs are not associated with gingival disease.

\section{RESULTS}

Altogether, 231 patients (119 female and 112 male) who gave written consent for the study were examined. Total of 1362 teeth were included in the study, among 
Journal of College of Medical Sciences-Nepal, 2014, Vol-10, No-1

which 456 were canines, 450 lateral incisors and 456 central incisors. Kappa test showed substantial interobserver and intra-observer agreement. The total prevalence of PGGs was $6.6 \%$ for maxillary anteriors. The prevalence of coronal PPGs was $15.7 \%$ for lateral incisors, $2.4 \%$ for canines and $1.5 \%$ for central incisors.

Coronal PPGs were more common in females $(56.6 \%)$ than in male $(43.3 \%)$ patients. Bilateralism was found in $26.6 \%$ of the patients. The details of frequency of PGGs and their location are given in table 1. Prevalence of radicular groove was only $0.88 \%$ for lateral incisors and $0.2 \%$ for canines. No radicular PGG was detected in maxillary central incisors.

As represented by table 2, Chi-square test did not show any significant association between PGGs and gingival disease $(\mathrm{P}=.91)$.

Table 1: Palato-gingival grooves and location in maxillary anteriors.

\begin{tabular}{lcccc} 
Tooth & $\begin{array}{c}\text { Total teeth } \\
\text { examined }\end{array}$ & Location & $\begin{array}{c}\text { Frequency } \\
\text { of PGGs }\end{array}$ & Prevalence \\
Central incisor & 456 & Mesial & 2 & \\
& & Mid-palatal & 4 & $1.5 \%$ \\
Lateral incisor & 450 & Disto-palatal & 1 & \\
& & Mesial & 10 & $15.7 \%$ \\
Canine & & Mid-palatal & 54 & \\
& 456 & Disto-palatal & 9 & $2.4 \%$ \\
Total & & Mesial & 1 & \\
\hline
\end{tabular}

Table 2: Presence of PGGs and gingival disease

\begin{tabular}{|c|c|c|c|c|}
\hline \multicolumn{5}{|c|}{ Presence of gingival disease } \\
\hline & & No & Yes & \\
\hline Presence of & No & 502 & 770 & 1272 \\
\hline PGG & Yes & 36 & 54 & 90 \\
\hline Total & & 538 & 824 & 1362 \\
\hline
\end{tabular}

$\mathrm{P}=0.91$ (Fisher's Exact Test) 


\section{DISCUSSION}

This study investigated the prevalence of coronal and radicular PGGs in patients visiting DOPD of Dhulikhel Hospital. PGG is a rare development anomaly leading to pulpal and periodontal diseases.

The tube-like channel of PGG serves as an ideal locus for plaque and calculus accumulation, thus acting as a secondary local etiologic factor, encouraging the development of periodontal disease. ${ }^{14}$ Its clinical and radiographic examination mimics endo-perio lesions leading to diagnostic dilemma, ineffective treatment attempts and treatment failures. Till date, no study has been done to investigate the prevalence of palatogingival grooves in Nepalese population. Therefore, it was important to investigate the prevalence of PPGs.

In this study, there was a prevalence of $6.6 \%$ of PGGs in maxillary anteriors. This was similar to studies done by $\mathrm{Kogan}^{5}$, which showed a prevalence of $4.6 \%$ among 3168 extracted maxillary incisors, and Pecora ${ }^{6}$, which showed a prevalence of three percent in lateral incisors of 642 patients. Study of Bacic et al. ${ }^{9}$ andWhiters et al. ${ }^{10}$ showed a lower prevalence of $1.01 \%$, two percent and $2.33 \%$ respectively.' However, the studies of PGGs on Libyan, Pakistani, Saudi and Brazilian population showed a higher prevalence of $7.5 \%, 10 \%, 10.3 \%$ and $11.1 \%$ respectively. ${ }^{3,7,8,12}$

These studies did not examine canines while our study included the canines as well. Few case reports have shown PGGs to be present in canines as well. Therefore the inclusion of canines for this study was essential and showed a prevalence of $2.4 \%$ which was higher than that of central incisors (1.5\%). Furthermore, this study used magnifying loupes which helped in detection of PGGs more accurately.
In this in-vivo study, the prevalence of radicular PGGs was only $0.88 \%$ for lateral incisors and $0.2 \%$ for canines. The lower prevalence may be because of low specificity of this observational study because the presence of radicular PGGs without loss of attachment may not have been detected with inspection and probing. Further study with the use of cone beam computer tomography (CBCT) and in-vitro studies are required for more accurate results.

This study showed no association between PGGs and gingival disease. However, results of Whiters et al. ${ }^{9}$ and Hou et al. ${ }^{11}$ showed that PGG is associated with poorer gingival health as measured by gingival index and more plaque accumulation as measured with plaque index. The study of Bacic et al. ${ }^{10}$ indicated that among 634 patients with periodontal disease, $0.75 \%$ had PGG. Similarly, higher percentage of bleeding was observed in cases with PGGs by Al-Sultan et al. ${ }^{2}$ Our study did not measure the amount of plaque accumulation. Therefore, we assume that gingival diseases in cases without PGG may be because of plaque accumulation which is may be associated with poor oral hygiene.

\section{CONCLUSION}

We can conclude that the prevalence of PGGs is high in our community and must be examined carefully to avoid diagnostic dilemmas. Gingival disease is not associated with palato-gingival groove.

\section{REFERENCES}

1. Ram S, Uma S, Pramod V, et al Palatogingival grooves. Indian Journal of Dental Advancements 2010;2(2):229-33. 
Journal of College of Medical Sciences-Nepal, 2014, Vol-10, No-1

2. Al-Sultan M, Al-Askar M, Al-Katheeri M, et al. The prevalence of the palatoradicular groove in a selected Saudi population and the effect on oral health.Saudi Dental Journal;21:125.

3. Santa Cecilia M, Lara VS, Moraes IG. The palato-gingival groove: a cause of failure in root canal treatment. Oral Surgery 1998;85:94-8.

4. Lara VS, Alberto C, Robert SB. Macroscopic and microscopic analysis of the palato-gingival groove. Journal of Endodontics 2000; 26(6): 345-50.

5. Kogon SL. The Prevalence, location and confirmation of palto radicular grooves in maxillary incisors. Journal of Periodontology 1986;57(4):231-4.

6. Pécora JD, da Cruz Filho AM. Study of the incidence of radicular grooves in maxillary incisors.Brazilian Dental Journal 1992;3(1):11-6.

7. Albaricci MF, de Toledo BE, Zuza EP, et al. Prevalence and features of palato-radicular grooves: an invitro study. Journal of International Academy of Periodontology 2008;10(1):2-5.

8. Iqbal N, Tirmazi SM, Majeed HA, et al. Prevalence of palato gingival groove in maxillary lateral incisors.Pakistan Oral and Dental Journal 2011;31(2):424-26.
9. Withers JA, Brunsvold MA, Killoy WJ, et al.The relationship of palato-gingival grooves to localized periodontal disease. Journal ofPeriodontology 1981;52(1):41-4.

10. Baciæ M, Karakas Z, Kaiæ Z, et al. The association between palatal grooves in upper incisors and periodontal complications.Journal of Periodontology 1990;61(3):197-9.

11. Hou GL, Tsai CC. Relationship between palatoradicular grooves and localized periodontitis. Journal of Clinical Periodontology 1993;20(9):678-82.

12. Radhakrishnan R, Deshpande N, Kasim SA. Prevalence of palatogingival grooves in a Libyan population and their relationship with periodontal diseases. Journal of Orofacial Research 2012;2(1):24-26.

13. Loe H, Silness J. The gingival index, the plaque index, and the retention index systems. Journal of Periodontology 1967;38:610-16.

14. Bose BB, Sudarsan S. Palatogingival groove an added dimension in the etiology of localised periodontitis. International Journal of Dental Science and Research 2013;1(1): 5-7. 